\title{
Full Electronic Structure Calculation of Quantum Mechanics in Binding Site of Protease 6LU7
}

\author{
Linxiang Zhou ${ }^{1}$ \\ ${ }^{1}$ Fudan University
}

September 26, 2020

\begin{abstract}
Here we report the binding site (We call it as drug target) of the COVID-19 main protease 3CLpro 6LU7 using Full Electronic Structure Calculation of Quantum Mechanics. The protease 6LU7 has 18038 elecrons, we need to calculate its wave funciton (it is called the molecular orbitor in the chemistry field) and energy level for this huge elecronic system. Then according to three rules to obtain the exact active pocket, active resudes, even active atoms of 6LU7. Finally put active pocket and all candidate small-molecule drugs or peptides group into an artificial intelligence deep learning 3D-CNN program to calculate the binding affinity. The result is that the pocket3 with three active atoms CE3, CZ3 and CH2 of 6LU7 is real design target for small-molecule drugs and the drug Tipranavir is located on the first position with binding affinity about 5.4. Our calculations show that the binding affinity of Remdesivir with 3CLpro 6LU7 is very small, less than 0.5. This just shows that Remdevivir (GS-5734) is the first channel. Because Remdesivir is not a nucleotide analog, it is a prodrug of nucleotide analogs, it has many protective groups around it. When the drug enters the body, these atomic groups will act as enzymes in the cell It is broken down, leaving the active drug and though the drug N3 company with 6 LU7, but its binding affinity is only about 4.2 , so it could not be a real drug for 6 LU7. The quantum mechanics calculation give out such accurate drug target. We have to take up pocket 3 to design inhibitor drug. The drug screening is only the first step in the long and costly pharmaceutical process, but we prove that it can be completely calculation by quantum mechanics. Hopefully, this method can provide an effective and rapid screening method for finding new coronavirus drugs.
\end{abstract}

\section{Hosted file}

Full Electronic Structure Calculation of Quantum Mechanics in Binding Site of Protease 6LU7.pdf available at https://authorea.com/users/362157/articles/483387-full-electronic-structurecalculation-of-quantum-mechanics-in-binding-site-of-protease-6lu7 